\title{
Successful treatment for infected biloma after endoscopic ultrasound-guided hepaticogastrostomy using double stent placement technique
}

\author{
Takeshi Ogura, Atsushi Okuda, Akira Miyano, Nobu Nishioka, Kazuhide Higuchi \\ Second Department of Internal Medicine, Osaka Medical College, Osaka 569-8686, Japan.
}

Correspondence to: Dr. Takeshi Ogura, Second Department of Internal Medicine, Osaka Medical College, 2-7 Daigakuchou, Takatsukishi, Osaka 569-8686, Japan. E-mail: oguratakeshi0411@yahoo.co.jp

How to cite this article: Ogura T, Okuda A, Miyano A, Nishioka N, Higuchi K. Successful treatment for infected biloma after endoscopic ultrasound-guided hepaticogastrostomy using double stent placement technique. Mini-invasive Surg 2018;2:8. http://dx.doi.org/10.20517/2574-1225.2017.48

Received: 24 Nov 2017 First Decision: 6 Mar 2018 Revised: 8 Mar 2018 Accepted: 29 Mar 2018 Published: 23 Apr 2018

Science Editor: Charles F. Bellows Copy Editor: Jun-Yao Li Production Editor: Huan-Liang Wu

\begin{abstract}
This case report describes a treatment of an elderly man who had undergone pancreaticoduodenectomy (Whipple Procedure) due to bile duct cancer. Herein, we describe technical tips of endoscopic ultrasound (EUS)-guided hepaticojejunostomy EUS-HJS combined with EUS-guided antegrade stenting (EUS-AS) using novel plastic stent. First, intrahepatic bile duct was punctured using $19 \mathrm{G}$ fine needle aspiration needle. Next, the 0.025-inch guidewire was inserted into the biliary tract. After the guidewire was advanced into the intestine, the bile duct and the intestine wall were dilated using by balloon catheter. The covered metal stent delivery system was antegradely inserted across the stricture site, and stent placement was performed from the intestine to the bile duct. Finally, stent placement from the intrahepatic bile duct to the intestine using novel plastic stent was successfully performed without any adverse events.
\end{abstract}

Keywords: Endoscopic ultrasound, endoscopic ultrasound-guided hepaticojejunostomy, liver abscess, endoscopic ultrasound-guided biliary drainage

\section{INTRODUCTION}

Trans-jejunum biliary drainage with endoscopic ultrasound (EUS)-guided hepaticojejunostomy (EUS-HJS) is now a well established procedure ${ }^{[1]}$. However, EUS-guided biliary drainage (EUS-BD) procedures have possibility of several adverse events such as stent migration or bile leakage. To prevent these adverse events, a covered, self-expandable, metal stent (CSEMS) is usually selected and EUS-BD is sometimes combined with EUS-guided antegrade stenting (AS). If CSEMS obstruction occurs, re-intervention is challenging in

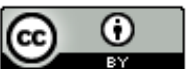

(C) The Author(s) 2018. Open Access This article is licensed under a Creative Commons Attribution 4.0 International License (https://creativecommons.org/licenses/by/4.0/), which permits unrestricted use, sharing, adaptation, distribution and reproduction in any medium or format, for any purpose, even commercially, as long as you give appropriate credit to the original author(s) and the source, provide a link to the Creative Commons license, and indicate if changes were made.

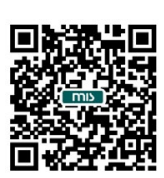



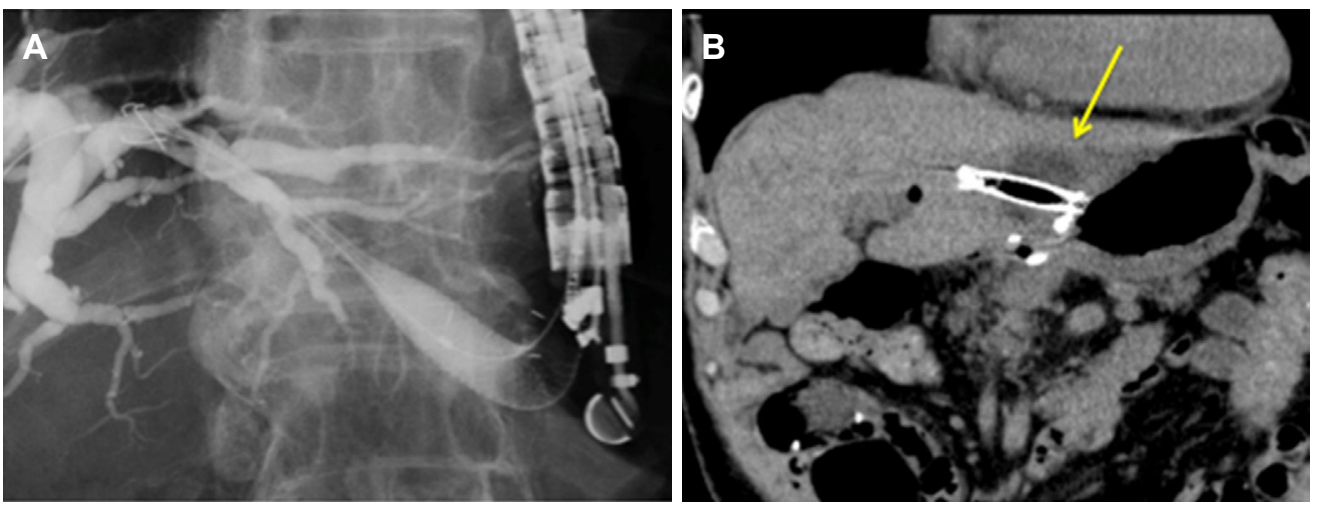

Figure 1. (A) Endoscopic ultrasound-guided hepaticogastrostomy using covered metal stent; (B) infected biloma is seen around endoscopic ultrasound-guided hepaticojejunostomy stent (arrow)

patients with a history of EUS-HJS because of limited lumen space compared with the stomach. Also, due to large diameter of EUS-HJS stent, bile juice reflux may cause vomiting. Recently, novel plastic stent has been introduced available in Japan. The plastic stent, which is a push-type stent and usually not possible to retract, has a total length of $20 \mathrm{~cm}$, an effective length of $15 \mathrm{~cm}$, and 4 flanges. The proximal end has a pigtail structure to prevent stent migration and the distal end is tapered ${ }^{[2,3]}$. This plastic stent has clinical impact because it is able to prevent stent migration into the abdominal cavity.

Although EUS-guided biliary drainage, such as hepaticogastrostomy (HGS), offers an alternative method to percutaneous transhepatic biliary drainage ${ }^{[2-5]}$, various adverse events such as stent migration into the abdominal cavity are associated with EUS-HGS and considerable effort has been directed towards preventing them ${ }^{[4,5]}$. Despite these efforts, adverse events such as infected biloma after EUS-HGS still arise due to frequent reflux cholangitis through EUS-HGS stent, or bile duct obstruction by a covered metal stent ${ }^{[6]}$. A longer HGS stent may help to prevent reflux cholangitis ${ }^{[5]}$, but if infected biloma occurs around an EUS-HGS, the stent must be exchanged. Other adverse events including liver abscess, may occur due to various reasons, and should be treated ${ }^{[7-11]}$. Here, we describe treatment of a liver abscess around an EUS-HGS, using double stent placement stent in an elderly man who had a history of surgery for bile duct cancer.

\section{CASE REPORT}

A 78-year-old man who had undergone pancreaticoduodenectomy due to bile duct cancer 1 year previously, was admitted to our hospital with obstructive jaundice. A benign bile-jejunum anastomosis stricture was diagnosed with computed tomography. Because the patient declined to undergo percutaneous drainage, the doctor proposed an alterative EUS-HGS procedure. The procedure was performed using a $10 \mathrm{~mm} \times 10 \mathrm{~cm}$, Niti-S Biliary Covered Stent (partially-covered, TaeWoong Medical, Seoul, South Korea; Century Medical Inc., Tokyo, Japan) [Figure 1A]. This resolved the obstructive jaundice, no adverse events occurred, and the patient was discharged after 2 weeks.

Four weeks after the EUS-HGS procedure, the patient presented with a fever and elevated inflammatory indicators and was consequently readmitted to hospital. Computed tomography revealed a biloma around the EUS-HGS stent [Figure 1B]. This biloma was considered to be complicated with infection. Endoscopic treatment for infected biloma was attempted as follows. An endoscopic retrograde cholangiopancreatography (ERCP) catheter (MTW Endoskopie, Düsseldorf, Germany) was initially inserted into the biliary tract through the EUS-HGS stent. A 0.025-inch guidewire (VisiGlide; Olympus Medical Systems, Tokyo, Japan) was inserted into the catheter and the metal stent was removed through 

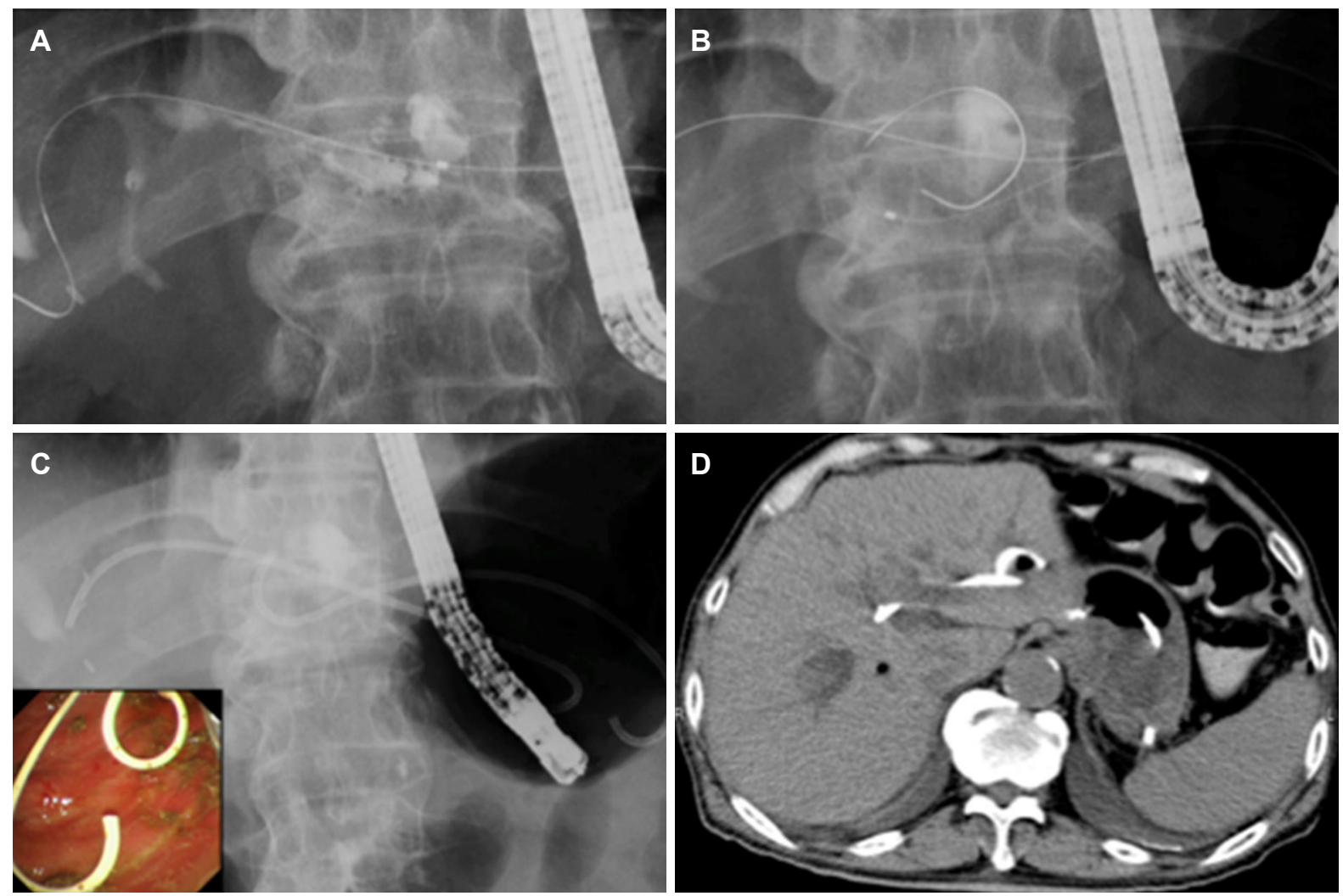

Figure 2. (A) Contrast-enhanced cholangioscopy image shows infected biloma; (B) guidewire placed in liver abscess and biliary tract; (C) deployed double stent; (D) computed tomography image after this procedure

the scope (JF 260V; Olympus Medical Systems, Tokyo, Japan). An additional ERCP catheter was inserted into the biliary tract, contrast medium was injected, and cholangiography visualized the infected biloma [Figure 2A]. Therefore, guidewires were inserted through the cateters in both the infected biloma and biliary tract [Figure $2 \mathrm{~B}$ ] so that a new EUS-HGS could be performed. A 7-Fr, double pig tail, 12-cm plastic stent (Medi-Globe GmbH; Achenmühle, Germany) was placed from the infected biloma to the stomach. Finally, a new, plastic stent placement (Type IT; Gadelius Medical Co, Ltd, Tokyo, Japan) was completed the EUS-HGS proedure [Figure $2 \mathrm{C}$ ].

Thereafter, after 1 week, inflammatory indicators and clinical symptoms were immediately resolved and the patient was discharged. This stent was removed after 2 months, and recurrence of biloma was not seen.

\section{DISCUSSION}

Fully covered metal stents deployed via EUS-HGS offer several advantages. Bile leakage from the gap between a fully covered metal stent and a fistula created during EUS-HGS to insert various devices may be less likely. This type of stent also remains patent for longer periods than plastic stents ${ }^{[11]}$. In addition, a fully covered metal stent itself can confer a tamponed effect on bleeding from the stomach wall or vessels around the bile duct. The disadvantages of metal stents include high cost, potential for branch bile duct obstruction, and the possibility of shortening. Focal cholangitis due to branch bile duct obstruction by a covered metal stent deployed after EUS-HGS is an adverse event that can usually be conservatively treated $^{[12]}$. However, a complicating, infected biloma is likely to require intervention. Kumata et al. ${ }^{[12]}$ described a hepatic abscess that developed within the cavity between the stomach and liver after EUSHGS. A $15-\mathrm{mm}$, lumen-apposing metal stent was deployed because the abscess could be accessed from 
the stomach and anchored by placing a plastic stent through it. The liver abscess in our patient was located in the hepatic parenchyma, which precluded the use of a lumen-apposing metal stent. Therefore, our technique is clinically useful for treating liver abscesses that arise after EUS-HGS, if percutaneous approach is refused by patients.

\section{DECLARATIONS}

\section{Authors' contributions}

Manuscript writing: Ogura T

Interpretation of data for the work, revising it critically for important intellectual content, final approval of the version to be published, agreement to be accountable for all aspects of the work in ensuring that questions related to the accuracy or integrity of any part of the work are appropriately investigated and resolved: Ogura T, Okuda A, Miyano A, Nishioka N, Higuchi K

\section{Financial support and sponsorship}

None.

\section{Conflicts of interest}

The authors declare that there are no conflicts of interest.

\section{Patient consent}

Obtained.

\section{Ethics approval}

Not applicable.

\section{Copyright}

(c) The Author(s) 2018.

\section{REFERENCES}

1. Nakai Y, Hamada T, Isayama H, Itoi T, Koike K. Endoscopic management of combined malignant biliary and gastric outlet obstruction. Dig Endosc 2017;29:16-25.

2. Dhir V, Isayama H, Itoi T, Almadi M, Siripun A, Teoh AYB, Ho KY. Endoscopic ultrasonography-guided biliary and pancreatic duct intervenetions. Dig Endosc 2017;29:472-85.

3. Sharaiha RZ, Khan MA, Kamal F, Tyberg A, Tombazzi CR, Ali B, Tombazzi C, Kahaleh M. Efficacy and safety of EUS-guided biliary drainage in comparison with percutaneous biliary drainage when ERCP fails: a systematic review and meta-analysis. Gastrointest Endosc 2017;85:904-14.

4. Cho DH, Lee SS, Oh D, Song TJ, Park DH, Seo DW, Lee SK, Kim MH. Long-term outcomes of a newly developed hybrid metal stent for EUS-guided biliary drainage (with videos). Gastrointest Endosc 2017;85:1067-75.

5. Ogura T, Yamamoto K, Sano T, Onda S, Imoto A, Masuda D, Takagi W, Fukunishi S, Higuchi K. Stent length is impact factor associated with stent patency in endoscopic ultrasound-guided heapticogastrostomy. $J$ Gastroenterol Hepatol 2015;30:1748-52.

6. Lin HF, Liao KF, Chang CM, Lin CL, Lai SW. Correlation between proton pump inhibitors and risk factor of pyogenic liver abscess. Eur J Clin Phamacol 2017;73:1019-25.

7. Liao KF, Lai SW, Lin CL, Chien SH. Appendectomy correlates with increased risk of pyogenic liver abscesss: a population-based cohort study in Taiwan. Medicine (Baltimore) 2016;95:e4015.

8. Liao KF, Cheng KC, Lin CL, Lai SW. Statin use correlates with reduced risk of pyogenic liver abscess: a population-based case-control study. Basic Clin Phamacol Toxicol 2017;121:144-9.

9. Lai SW, Lin CL, Liao KF. Association between oral corticosteroid use and pyogenic liver abscesses in a case-control study. Biomedicine (Taipei) 2018;8:5.

10. Mei-Ling S, Kuan-Fu L, Sung-Mao T, Cheng-Li LM, Shih-Wei L. Herpes zoster correlates with pyogenic liver abscesses in Taiwan. Biomedicine (Taipei) 2016;6:22.

11. Ogura T, Higuchi K. Technical tips for EUS-guided hepaticogastrostomy. World J Gastroenterol 2016;22:3945-51.

12. Kumata NA, Torres-Ruiz F, Reinosno PJ, Kahaleh M. Endoscpic management of hepatic abscesss after EUS-guided hepaticogastostomy. Gastrointest Endosc 2016;84:1054-5. 